\title{
In silico trials of food digestion and absorption: how far are we? Steven Le Feunteun ${ }^{1}$, Alan Robert Mackie ${ }^{2}$ and Didier Dupont ${ }^{1}$
}

In recent years, experimental research on the mechanisms of food digestion in the gastrointestinal tract has strengthened our knowledge on the effect of food on human health. A number of mathematical models have been proposed to rationalize our understanding on the related mechanisms. One common suggestion is that in silico models could be interconnected and used in the future to predict the effect of food systems (liquid or solid, inner microstructure, state of nutrients ... ) on various metabolic responses. This paper aims to provide a brief overview of the latest developments in this young but promising field of research.

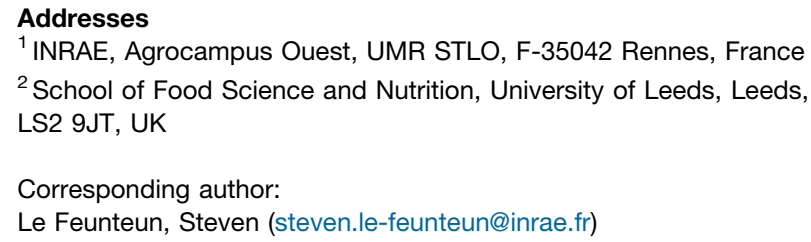

\section{Current Opinion in Food Science 2020, 31:121-125}

This review comes from a themed issue on Food chemistry and biochemistry

Edited by Uri Lesmes

https://doi.org/10.1016/j.cofs.2020.04.006

2214-7993/@ 2020 Elsevier Ltd. All rights reserved.

\section{Introduction}

The increase in diet-related diseases has fuelled the need to improve our knowledge on the fate of foods, or meals, in the gastrointestinal (GI) tract. Over the past 15 years, research in this field has provided new insights into why and how the structure of the food we eat can affect the kinetics and extent of nutrient absorption [1,2]. As with pharmaceuticals, the dose and timing of nutrient arrival in the blood stream during digestion have important metabolic consequences. Examples include both deleterious and beneficial repercussions, as for instance the increased risk of type-2 diabetes for diets with high glycaemic index [3], or the stimulation of protein muscle synthesis above a threshold of leucine in the peripheral blood [4]. To advance further in our understanding of the relationship between foods, or diets, with the overall functioning of the human gastrointestinal (GI) tract and post-absorptive processes, systems modelling of digestion appears as a promising means. Such an approach can be used to tackle the diversity of the mechanisms that take place during digestion, and could be very valuable for predictive purposes. This has already happened in the pharmaceutical area, in which the concept of 'in silico clinical trials' has emerged as a new tool in the drug regulatory process $[5,6]$. This paper therefore questions the possibility of advancing towards establishing models of food digestion and nutrient absorption that could help predict the metabolic responses to foods and meals. It starts by describing some attempts that have been proposed in the past, before reviewing the latest developments and the remaining challenges related to this field of research.

\section{Physiologically based kinetic (PBK) modelling of food digestion: where do we stand?}

Predictive models of food's GI transit, hydrolysis and absorption can be built with classical engineering approaches, in which the digestive system is split into a number of anatomical compartments (e.g. one or several gastric and intestinal sub-compartments, peripheral blood, etc.) and into a series of unit operations to model the physicochemical processes that take place $\left[7^{0^{\bullet \bullet}}\right]$. In the pharmaceutical area, this strategy has led to a number of physiologically based kinetic (PBK) models to predict the absorption of orally administered pharmaceuticals [5], or for safety assessment of chemicals: cosmetics, food additives, pesticides, and so on [8]. Thanks to their capability to predict the overall internal exposure to a chemical and on its ability to elicit a biological response, these models are becoming more and more essential before in vivo experiments can be undertaken.

The same principles can be applied to relate some nutritional considerations (e.g. extent and kinetics of nutrient absorption) with food or meal digestion. PBK models of the GI transit and absorption of meals, from stomach to colon with consideration of all kinds of macronutrients, were proposed for pigs quite a long time ago $[9,10]$. The latest version of this model [10] seemed to accurately predict the digestibility of the main food components, lipid excepted, as well as the absorption profile of the studied nutrients (glucose, amino acids, and volatile fatty acids) over a large variety of diets. The same strategy could thus be adapted for human physiology. One lack, however, is that they do not directly account for the physical properties of ingested foods or meals. Hence, these are not directly suitable to predict food structure effects. In another study, a PBK model dedicated to dairy 
protein digestion and absorption in mini-pigs showed that the great differences in the kinetics of amino acid absorption experimentally observed for differently structured dairy matrices of identical composition could be fairly reproduced by considering contrasting gastric behaviours and emptying kinetics [11]. By distinguishing the fraction of gastric content that is ready to be emptied into the small intestine from the one that is not ready yet (e.g. large food particles), this model provided a first attempt to integrate food structure considerations into PBK models of food digestion.

These examples are interesting in that they can be used to predict the dose and timing of nutrient arrival in the blood stream in response to a given food or a meal, and could be easily connected with nutritional models that aim to predict the metabolic fate and consequences of absorbed amino-acids [12], lipid products [13], or sugars [14] in the fed state. However, to build a PBK model of food transit and absorption that becomes truly relevant for predictive purposes, there remains a clear need to more directly relate the properties of the foods or meals with: (i) the kinetics of enzymatic hydrolysis, and (ii) the GI transit, in particular the gastric emptying kinetics. As further reviewed, a number of models are now becoming available to tackle these issues.

\section{How to account for key properties of macronutrients in the modelling of enzymatic hydrolysis and GI transit?}

Current developments in the modelling of enzymatic hydrolysis mostly originate from the ongoing efforts to take into account the key properties of the main nutrients (i.e. proteins, lipids and starch). In this field, we may shed light on the results recently obtained from approaches that consider multiple species within each type of macronutrient, rather than trying to model an average behaviour. For instance, a model accounting for the fatty acid composition of an oil-in-water emulsion, and for rate constants that are specific for each fatty acid residue, not only allowed a better fitting of the in vitro disappearance of triacylglycerols but also proved very efficient in predicting the individual bioaccessibility profiles of each oil's fatty acid [15]. Another multi-response model has been successfully applied to the hydrolysis profiles of the main lipid categories (triglycerides, monoglycerides, and free fatty acids) for a total of 28 different data sets of emulsion intestinal in vitro digestions [16]. Comparable approaches have also been proposed to model the hydrolysis kinetics of proteins [17,18], and starch [19-21], where consideration is made that different rates of hydrolysis can be assumed for different subclasses of the considered substrate, that is, more or less resistant and/or accessible fractions. These examples are of particular interest because they provide a direct means of incorporating both compositional and structural information in enzymatic hydrolysis models, with non-empirical relations between the model parameters and the food properties. Since they all rely on the same principles and are relatively simple to build or modify, these approaches could thus be assembled and interconnected to enable predictive modelling of the release kinetics of all kinds of nutrient from food material(s). The large body of experimental data already existing in the literature could be used to identify the most appropriate model structures and tune their parameterization.

Gastric emptying is another key determinant of the overall nutrient absorption kinetics. It is well known that this process is, in first approximation, governed by a feedback mechanism controlling the flux of calories delivered to the proximal small intestine. Indeed, it was shown more than 40 years ago that gastric emptying of meals can be fairly predicted from the meal's initial volume and caloric density [22]. More recently, Moxon et al. proposed a gastric emptying model for nutrient liquid meals that include a nutrient feedback mechanism, and which further takes into account the variations of the chyme viscosity upon gastric secretions and emptying $\left[23^{\circ}\right]$. Overall, this model closely simulated in vivo gastric emptying patterns of liquid meals varying by both their nutrient content and viscosity. Although the predictive capability of this model needs to be validated further, it certainly constitutes a much more elegant basis than mass action laws or empirical equations to predict the gastric emptying of liquid meals in PBK models of food digestion. Overall, almost all pieces seem now available to develop PBK models that could predict the main nutritional responses to liquid foods.

\section{Three-dimensional computational models of food breakdown and mixing}

Food is not simply a soup of nutrients. It has structure that is complex and multiscale, requiring phenomena occurring at a larger scale to be taken into account for solid foods. The comminution of food and mixing with GI fluids are primarily important at the oral and gastric stages, where food pieces are larger and less diluted by the digestive secretions. Food fragment size and structure may not only impact enzymatic hydrolysis but also gastric emptying, which largely controls the overall kinetics of nutrient uptake [24]. It is thus important to develop models that could predict food fragmentation and mixing in the upper part of the GI tract. These mechanisms are generally simulated using computational solid mechanics and fluid dynamics.

During the oral phase, solid foods are broken down and lubricated with saliva. Food oral processing therefore governs the size distribution of food particles that reach the stomach, which are typically up to several millimetres in size. To enhance our understanding of the relationship between food structure and oral breakdown during mastication, latest results from both finite element [25] 
and meshfree [26,27] three-dimensional (3D) methods are rather encouraging. When combined with experimental data from model food materials, they enabled fair predictions of crack initiation and propagation [25], and of food fragment sizes produced during chewing [26]. This latter model, proposed by Harrison et al., used smoothed particle hydrodynamics (SPH) to predict the mechanical behaviour and breakdown of two agar gels during mastication. It is particularly interesting in that several chewing cycles are considered and the predicted fracture damage and particle size distributions are directly related to the measured properties of their food materials. These computational models still remain in their early stages but seem extendable to a variety of food structures with different rheological properties. In the future, it is therefore possible that these approaches could be used to predict fragment sizes of oral bolus from known or measured food properties and anatomical considerations.

At the gastric stage, pioneer 3D computational models of the entire stomach date from the early 2010s, by Ferrua and Singh [28,29] and Imai et al. [30]. These models considered the case of a closed pylorus to investigate gastric mixing of liquid meals of different viscosity. More recently, Harrison et al. have also developed an SPH-based model to simulate gastric mixing and emptying of aqueous solutions when the pylorus remains fully open [31], and the team of Imai have just investigated how the coordination between pyloric closure and antral contraction affects the emptying of liquid contents [32]. By considering gastric emptying, these new models represent an important step forward in improving our understanding of gastric digestion. However, despite their great merits, current gastric computational models do not yet account for secretions and enzymatic reaction(s) and have only focused on liquids, with no or few discrete solid particles. Several important developments thus remain to be performed before a comprehensive multiphysics model of the stomach can be used to predict the gastric breakdown, mixing and emptying of solid foods $\left[33^{\circ}\right]$.

Peristatic and segmentation contractions in the small intestine have also been simulated with computational fluid dynamics to predict the flow and mixing at different length scales [34-37]. These studies have notably shown that the transport of nutrients from the intestinal lumen to the wall can be significantly reduced when the apparent viscosity of digesta is high [34], and that the motion of villi at the gut wall can significantly enhance the mixing in the proximity of epithelium cells [35-37]. Overall, these works have provided important insights to better understand the rate limiting steps of nutrient absorption, and on the possible effect of the mechanical properties of food.

Computational modelling of food breakdown and mixing in the GI tract has already provided important findings. If more work remains to be performed, in particular at the oral and gastric stages, this field of research is still young and ongoing developments in numerical methods rapidly improve the accuracy and speed of complex simulation scenarios. It is therefore expectable that these approaches will become more and more accurate and reliable in the forthcoming years.

\section{Alternative approaches to predict the breakdown and transit of solid foods}

In relation to the objective of predicting the main metabolic responses to a food or a meal from in silico modelling, it may not always be necessary to simulate the 3D-spatiotemporal evolution of the GI content. Predictions of food transit in one spatial dimension (i.e. along the GI tract) should be adequate for most nutritional considerations. From a systems modelling perspective, as in PBK models, this strategy also facilitates model computations and interconnections.

A number of research groups continue to use and adapt models developed in the fields of engineering and biophysics to predict the behaviour of food in gastric conditions $\left[7^{\bullet \bullet}\right]$. Recent examples include modelling work on mass transfer and absorption in the intestine [38], or on the physical-chemistry of gastric digestion of solid foods to predict: their swelling [39], their softening [40], their breakdown into particles [41], their acid and moisture uptake and buffering capacity [42-44]. Recently, a more integrative approach has also been proposed by Sicard $e t a l$. to model of the gastric digestion of meat proteins $\left[45^{\circ \bullet}\right]$. They built a reaction-diffusion model that accounts for a number of mechanisms: pepsin and proton diffusion in bolus particles, pepsin activity as a function of $\mathrm{pH}$, the buffering capacity of meat, and indirectly for the movement of particles with secretion via a mass transfer coefficient at the meat particle-gastric fluid interface. As discussed by the authors, the current version of their model still has room for improvement, in particular by computing the progressive reduction of the particle size in relation to gastric emptying kinetics. Because the structure of this model has a very general character, this work appears suitable to be transposed to other types of solid foods, meanwhile it could also be integrated into PBK models of food digestion and absorption. In the future, further developments might enable prediction of the gastric digestion and emptying of solid foods from physical considerations in place of the classically employed empirical equations. This represents the main modelling challenge to overcome before predictive models of solid food digestion and absorption could become a reality.

\section{The essential role of experimental data}

Another bottleneck in establishing a comprehensive model of food digestion is the need to rely on relevant experimental data and knowledge. Because in vitro experiments use wellcontrolled conditions, they provide a very good framework to test some modelling assumptions, in particular with regards to the effects of food composition and structure on enzymatic and disintegration kinetics. Recent reviews have also discuss the ability and limits of both static [46] and dynamic [47] 
in vitro experiments to reproduce in vivo observations. Nonetheless, in vitro experiments do not reflect the reality of in vivo digestion, which is regulated by both neural and hormonal feedback mechanisms.

Hence, to develop in silico models of food digestion that become physiologically relevant, there is a clear need to rely on in vivo data, and more particularly on human data whenever possible. However, most of the previously described models rely on a small sample of the physiology literature. Since there is no in vivo database that modellers could use to build and evaluate their models, one of the main challenges they face is to find, extract and assess the relevant quantities from articles across various scientific fields. This is complicated by the fact that modellers do not always have a background in animal or human digestion physiology; meanwhile, experts in physiology and metabolism are not necessarily aware of the needs and constraints of modelling. To advance, the communities of experimentalists and modellers will have to collaborate more closely to identify and gather relevant experimental data sets and knowledge for model development and evaluation. In this context, modelling should also serve as a knowledge-based system, in which our understanding of the mechanisms and of their relationships is organized and can be incrementally improved and complete.

\section{Conclusion}

Modelling the digestive processes is challenging and research in this area is currently very active. The scope of existing models spread from molecular mechanisms up to systems view approaches with increasing efforts to relate food properties to key mechanisms of food digestion. Previously proposed PBK models of food digestion and absorption could largely benefit from recent advances in the modelling of food structure effects on digestion, from the enzymatic hydrolysis of macronutrients up to the impact of food macrostructure. All the pieces seem now available to start building in silico models that could predict the main metabolic responses to liquid foods and meals, although more work remains to be done for the case of solid foods.

\section{Conflict of interest statement}

Nothing declared.

\section{References and recommended reading}

Papers of particular interest, published within the period of review, have been highlighted as:

- of special interest

•• of outstanding interest

1. Dupont D, Le Feunteun S, Marze S, Souchon I: Structuring food to control its disintegration in the gastrointestinal tract and optimize nutrient bioavailability. Innov Food Sci Emerg Technol 2018, 46:83-90.

2. Gouseti O, Bornhorst G, Bakalis S, Mackie A (Eds): Interdisciplinary Approaches to Food Digestion. Springer International Publishing; 2019.
3. Bhupathiraju SN, Tobias DK, Malik VS, Pan A, Hruby A, Manson JE, Willett WC, Hu FB: Glycemic index, glycemic load, and risk of type 2 diabetes: results from 3 large US cohorts and an updated meta-analysis. Am J Clin Nutr 2014, 100:218-232.

4. Rieu I, Balage M, Sornet C, Giraudet C, Pujos E, Grizard J, Mosoni L, Dardevet D: Leucine supplementation improves muscle protein synthesis in elderly men independently of hyperaminoacidaemia. J Physiol 2006, 575:305-315.

5. Zhuang $X$, Lu C: PBPK modeling and simulation in drug research and development. Acta Pharm Sin B 2016, 6:430-440.

6. Pappalardo F, Russo G, Tshinanu FM, Viceconti M: In silico clinical trials: concepts and early adoptions. Brief Bioinf 2019 , 20:1699-1708 http://dx.doi.org/10.1093/bib/bby043 https://www. ncbi.nlm.nih.gov/pubmed/29868882.

7. Lamond AR, Janssen AEM, Mackie A, Bornhorst GM, Bakalis S, -. Gouseti O: An engineering perspective on human digestion. In Interdisciplinary Approaches to Food Digestion. Edited by Gouseti O, Bornhorst GM, Bakalis S, Mackie A. Springer International Publishing; 2019:255-273.

This book chapter reviews the mechanics of human digestion from an engineering viewpoint. It provides an engineering analysis of the gut, which includes dimensional analysis and identification of the key parameters and phenomena that determine the rate and extent of food digestion. The use of mathematical models and computational fluid dynamics are also discussed for the study of digestive processes, in particular gastric and intestinal flow and mixing.

8. Madden JC, Pawar G, Cronin MTD, Webb S, Tan Y-M, Paini A: In silico resources to assist in the development and evaluation of physiologically-based kinetic models. Comput Toxicol 2019, 11:33-49.

9. Bastianelli D, Sauvant D, Rérat A: Mathematical modeling of digestion and nutrient absorption in pigs. J Anim Sci 1996, 74:1873-1887.

10. Strathe AB, Danfær A, Chwalibog A: A dynamic model of digestion and absorption in pigs. Anim Feed Sci Technol 2008, 143:328-371.

11. Le Feunteun S, Barbe F, Remond D, Menard O, Le Gouar Y, Dupont D, Laroche B: Impact of the dairy matrix structure on milk protein digestion kinetics: mechanistic modelling based on mini-pig in vivo data. Food Bioprocess Technol 2014 , 7:1099-1113.

12. Fouillet H, Juillet B, Gaudichon C, Mariotti F, Tome D, Bos C: Absorption kinetics are a key factor regulating postprandial protein metabolism in response to qualitative and quantitative variations in protein intake. Am J Physiol Regul Integr Comp Physiol 2009, 297:R1691-R1705.

13. Jelic K, Hallgreen CE, Colding-Jørgensen M: A model of NEFA dynamics with focus on the postprandial state. Ann Biomed Eng 2009, 37:1897.

14. Rozendaal YJ, Maas AH, van Pul C, Cottaar EJ, Haak HR, Hilbers PA, van Riel NA: Model-based analysis of postprandial glycemic response dynamics for different types of food. Clin Nutr Exp 2018, 19:32-45.

15. Giang TM, Gaucel S, Brestaz P, Anton M, Meynier A, Trelea IC, Le Feunteun S: Dynamic modeling of in vitro lipid digestion: individual fatty acid release and bioaccessibility kinetics. Food Chem 2016, 194:1180-1188.

16. Verkempinck SHE, Salvia-Trujillo L, Infantes Garcia MR, Hendrickx ME, Grauwet T: From single to multiresponse modelling of food digestion kinetics: the case of lipid digestion. J Food Eng 2019, 260:40-49.

17. Barros RM, Xavier Malcata F: A kinetic model for hydrolysis of whey proteins by cardosin A extracted from Cynara Cardunculus. Food Chem 2004, 88:351-359.

18. Kondjoyan A, Daudin J-D, Santé-Lhoutellier V: Modelling of pepsin digestibility of myofibrillar proteins and of variations due to heating. Food Chem 2015, 172:265-271.

19. Li H, Dhital S, Gidley MJ, Gilbert RG: A more general approach to fitting digestion kinetics of starch in food. Carbohydr Polym 2019, 225:115244. 
20. Edwards $\mathrm{CH}$, Warren FJ, Milligan PJ, Butterworth PJ, Ellis PR: A novel method for classifying starch digestion by modelling the amylolysis of plant foods using first-order enzyme kinetic principles. Food Funct 2014, 5:2751-2758.

21. Meraz M, Alvarez-Ramirez J, Vernon-Carter EJ, Reyes I, Hernandez-Jaimes C, Martinez-Martinez F: A two competing substrates Michaelis-Menten kinetics scheme for the analysis of in vitro starch digestograms. Starch 2019:1900170.

22. Hunt JN, Stubbs DF: The volume and energy content of meals as determinants of gastric emptying. J Physiol 1975 245:209-225.

23. Moxon TE, Nimmegeers P, Telen D, Fryer PJ, Van Impe J,

- Bakalis S: Effect of chyme viscosity and nutrient feedback mechanism on gastric emptying. Chem Eng Sci 2017, 171:318-330.

This article presents a mathematical model for the gastric emptying of liquid foods that relies on a nutrient feedback mechanism in the proximal small intestine. Although this idea is not new (e.g. Ref. [22]), this study is probably the first in recent years to explicitly build a gastric emptying model upon this assumption. The model presented provided good fits to experimental data for both high-viscosity and low-viscosity liquid meals with high-nutrient and low-nutrient content.

24. Weber E, Ehrlein H-JR: Relationships between gastric emptying and intestinal absorption of nutrients and energy in mini pigs. Dig Dis Sci 1998, 43:13.

25. Skamniotis CG, Elliott M, Charalambides MN: On modeling the large strain fracture behaviour of soft viscous foods. Phys Fluids 2017, 29:121610.

26. Harrison SM, Eyres G, Cleary PW, Sinnott MD, Delahunty C, Lundin L: Computational modeling of food oral breakdown using smoothed particle hydrodynamics. J Texture Stud 2014 45:97-109.

27. Harrison SM, Cleary PW, Eyres G, Sinnott M D, Lundin L: Challenges in computational modelling of food breakdown and flavour release. Food Funct 2014, 5:2792-2805.

28. Ferrua MJ, Singh RP: Modeling the fluid dynamics in a human stomach to gain insight of food digestion. J Food Sci 2010, 75: R151-R162.

29. Ferrua MJ, Singh RP: Understanding the fluid dynamics of gastric digestion using computational modeling. Procedia Food Sci 2011, 1:1465-1472.

30. Imai Y, Kobayashi I, Ishida S, Ishikawa T, Buist M, Yamaguchi T: Antral recirculation in the stomach during gastric mixing. Am J Physiol Gastrointest Liver Physiol 2013, 304:G536-G542.

31. Harrison SM, Cleary PW, Sinnott MD: Investigating mixing and emptying for aqueous liquid content from the stomach using a coupled biomechanical-SPH model. Food Funct 2018, 9:3202-3219.

32. Ishida S, Miyagawa T, O'Grady G, Cheng LK, Imai Y: Quantification of gastric emptying caused by impaired coordination of pyloric closure with antral contraction: a simulation study. J R Soc Interface 2019, 16:20190266.

33. Brandstaeter S, Fuchs SL, Aydin RC, Cyron CJ: Mechanics of the - $\quad$ stomach: a review of an emerging field of biomechanics GAMM Mitteilungen 2019, 42:e201900001.

This review sums-up our current understanding of the mechanics of the human stomach and delineates the challenges in mathematical and computational modelling that remain to be addressed in this emerging field. It also illustrates potential applications of a computational multiphysics model of the human stomach in areas ranging from medicine to food industries.
34. Love RJ, Lentle RG, Asvarujanon P, Hemar Y, Stafford KJ: An expanded finite element model of the intestinal mixing of digesta. Food Dig 2013, 4:26-35.

35. de Loubens C, Lentle RG, Hulls C, Janssen PWM, Love RJ, Chambers JP: Characterisation of mixing in the proximal duodenum of the rat during longitudinal contractions and comparison with a fluid mechanical model based on spatiotemporal motility data. PLoS One 2014, 9:e95000.

36. Wang Y, Brasseur JG, Banco G, Webb AG, Ailiani AC, Neuberger T: A multiscale lattice Boltzmann model of macroto micro-scale transport, with applications to gut function. Philos Trans $R$ Soc A 2010, 368:2863-2880

37. Lentle RG, Janssen PWM, DeLoubens C, Lim YF, Hulls C, Chambers P: Mucosal microfolds augment mixing at the wall of the distal ileum of the brushtail possum. Neurogastroenterol Motil 2013, 25 881-e700.

38. Moxon TE, Gouseti O, Bakalis $\mathrm{S}$ : In silico modelling of mass transfer \& absorption in the human gut. J Food Eng 2016, $176: 110-120$.

39. van der Sman RGM, Houlder S, Cornet S, Janssen A: Physical chemistry of gastric digestion of proteins gels. Curr Res Food Sci 2020, 2:45-60 http://dx.doi.org/10.1016/j.crfs.2019.11.003.

40. Drechsler KC, Bornhorst GM: Modeling the softening of carbohydrate-based foods during simulated gastric digestion. J Food Eng 2018, 222:38-48.

41. Drechsler KC, Ferrua MJ: Modelling the breakdown mechanics of solid foods during gastric digestion. Food Res Int 2016, 88:181-190

42. Luo Q, Zhan W, Boom RM, Janssen AEM: Interactions between acid and proteins under in vitro gastric condition - a theoretical and experimental quantification. Food Funct 2018 9:5283-5289.

43. Mennah-Govela YA, Keppler S, Januzzi-Guerreiro F et al.: Acid and moisture uptake into red beets during in vitro gastric digestion as influenced by gastric pH. Food Biophys $2020 \mathrm{http}: / /$ dx.doi.org/10.1007/s11483-019-09623-w.

44. Mennah-Govela YA Singh RP, Bornhorst GM: Buffering capacity of protein-based model food systems in the context of gastric digestion. Food Funct 2019, 10:6074-6087.

45. Sicard J, Mirade P-S, Portanguen S, Clerjon S, Kondjoyan A:

- Simulation of the gastric digestion of proteins of meat bolus using a reaction-diffusion model. Food Funct 2018 , 9:6455-6469.

This study presents a reaction-diffusion mathematical model that has been developed to predict the gastric digestion of meat proteins. It is one of the most advanced attempt to model the gastric hydrolysis of a solid food by the number of mechanisms it takes into account: pepsin and proton diffusion in bolus particles, the buffering capacity of the meat, and the pepsin activity as a function of $\mathrm{pH}$. This paper not only reports simulation work predicting the effects of pepsin concentration, $\mathrm{pH}$ variations and meat particle size, but also lists several possible improvements, especially in the way it accounts for the time-course change in particle size.

46. Bohn T, Carriere F, Day L, Amelie D, Egger L, Freitas D, Golding M Le Feunteun S, Macierzanka A, Menard O et al.: Correlation between in vitro and in vivo data on food digestion. What can we predict with static in vitro digestion models? Crit Rev Food Sci Nutr 2018, 58:2239-2261.

47. Dupont D, Alric M, Blanquet-Diot S, Bornhorst G, Cueva C Deglaire A, Denis S, Ferrua M, Havenaar R, Lelieveld J et al.: Can dynamic in vitro digestion systems mimic the physiological reality? Crit Rev Food Sci Nutr 2019, 59:1546-1562. 